\title{
Nonlinear localized modes in dipolar Bose-Einstein condensates in two-dimensional optical lattices
}

\author{
Santiago Rojas-Rojas ${ }^{a, b, c, *}$, Uta Naether ${ }^{d}$, Aldo Delgado ${ }^{a, b, c}$, Rodrigo A. Vicencio ${ }^{a, b, e}$ \\ a Center for Optics and Photonics, Universidad de Concepción, Casilla 160-C, Concepción, Chile \\ ${ }^{\mathrm{b}}$ MSI-Nucleus on Advanced Optics, Universidad de Concepción, Casilla 160-C, Concepción, Chile \\ ' Departamento de Física, Universidad de Concepción, Casilla 160-C, Concepción, Chile \\ d Instituto de Ciencia de Materiales de Aragón and Departamento de Física de la Materia Condensada, CSIC-Universidad de Zaragoza, 50009 Zaragoza, Spain \\ e Departamento de Física, Facultad de Ciencias, Universidad de Chile, Santiago, Chile
}

\section{A R T I C L E I N F O}

\section{Article history:}

Received 1 February 2016

Received in revised form 14 May 2016

Accepted 12 July 2016

Available online $\mathrm{xxxx}$

Communicated by A.P. Fordy

\section{Keywords:}

Dipolar BECs

Discrete breathers

Peierls-Nabarro surfaces

\begin{abstract}
A B S T R A C T
We analyze the existence and properties of discrete localized excitations in a Bose-Einstein condensate loaded into a periodic two-dimensional optical lattice, when a dipolar interaction between atoms is present. The dependence of the Number of Atoms (Norm) on the energy of solutions is studied, along with their stability. Two important features of the system are shown, namely, the absence of the Norm threshold required for localized solutions to exist in finite 2D systems, and the existence of regions in the parameter space where two fundamental solutions are simultaneously unstable. This feature enables mobility of localized solutions, which is an uncommon feature in 2D discrete nonlinear systems. With attractive dipolar interaction, a non-trivial behavior of the Norm dependence is obtained, which is well described by an analytical model.
\end{abstract}

(c) 2016 Elsevier B.V. All rights reserved.

\section{Introduction}

The well-studied field of Bose-Einstein condensation has been expanded in recent years by the experimental realization of condensates whose atoms exhibit a magnetic moment, i.e., magnetic dipolar atoms. These experiments have been performed with Chromium atoms [1] and, more recently, Dysprosium atoms [2,3]. In these Bose-Einstein condensates (BECs), the interaction between dipoles has a long range dependence and $d$-wave symmetry. So, it is anisotropic with respect to the orientation of the dipoles and the separation between them [4,5]. The effect of this dipolar type of interaction on the properties of localized excitations (so-called breathers or solitons [6]) has attracted considerable interest since these were predicted in the one-dimensional (1D) [7-13] and twodimensional (2D) $[4,5,14]$ cases. In addition to long-range interactions and local two-body collisional contacts (characteristic of cold quantum gases), some authors have proposed to consider threebody collisions in dipolar gases, which allows for the observation of supersolid states [15]. Although the experimental observation of dipolar solitons has yet to be realized, a realistic setup for a 2D BEC was recently proposed in [16].

\footnotetext{
* Corresponding author at: Departamento de Física, Universidad de Concepción, Casilla 160-C, Concepción, Chile.

E-mail address: srojas@cefop.cl (S. Rojas-Rojas).
}

When a BEC is loaded into a deep periodic optical potential, the resulting system can be described by a nonlinear discrete (lattice) model. If only short range collisional interactions are present, an appropriate model is the Discrete Nonlinear Schrödinger Equation (DNLS), a differential equation governing the amplitude of the wavefunction at each site of the lattice, A cubic-nonlinear term $[17,18]$ models the interaction effectively. In this case, an interesting feature appears regarding the existence of localized solutions in two-dimensional lattices: for a DNLS model with linear interactions and local nonlinearities, there is a minimum value for the total number of particles composing the BEC solution (the so-called Norm), below which localized discrete solutions cannot exist [19] in infinite lattices. This Norm threshold implies that a localized BEC cannot be excited below a minimum number of atoms in a 2D optical lattice [20]. The DNLS equation can be modified by introducing different linear or nonlinear coupling terms, which may affect the Norm threshold. For instance, it has been shown that in the Ablowitz-Ladik model the nonlinear coupling terms decrease this threshold, but it only ceases to exist for very small lattice sizes [21]. When linear dipolar interactions are included [22], the linear coupling between sites could become anisotropic, affecting also the Norm threshold and the stability of localized solutions.

Another feature of nonlinear two-dimensional lattices is that mobility of localized solutions is rare or very difficult. For cubic local nonlinearities only broad modes are able to overcome the ef- 
fective energy barrier and move across the lattice $[23,24]$. This is due to the fact that fundamental broad solutions possess almost no geometrical distinction. The values of their Hamiltonians are nearly identical and, therefore, the Peierls-Nabarro potential [25] is essentially zero. Localized fundamental modes, which occupy only few lattice sites, are forbidden to move in this context and simply decay into radiation when it is tried due to the large PeierlsNabarro barrier they have to overcome. On the other hand, for a saturable nonlinearity (also for cubic-quintic systems [26]) solutions get broader while increasing the Norm, and several Hamiltonian crossings between different fundamental solutions occur. This generates repeated mobility windows [27,28], where mobility of highly localized solutions occurs due to the appearance of additional asymmetric intermediate solutions. A recent review on mobility in discrete nonlinear lattices can be found in Ref. [29]. Based on recent results on dipolar one-dimensional lattices [30], showing similar saturation features, a good mobility may be expected for 2D dipolar lattices as well.

In Ref. [14], a general discrete model to study breathers in 2D dipolar BECs was derived and analyzed in certain parameter space regions. However, some very fundamental properties of this system still need to be studied in detail, as the dependence on the system parameters for the Norm threshold and the mobility of localized solutions. In this work, we study the properties of localized modes in a dipolar BEC loaded into a 2D optical lattice by using a generalization of the Discrete Nonlinear Schrödinger Equation. Our model allows isotropic nonlinear coupling between sites in the form proposed in Ref. [30] for 1D lattices. A stability exchange between three different fundamental solutions is observed, which leads to the existence of asymmetric (or intermediate) stationary solutions. We address the study of the mode properties by computing an effective dynamical potential, which proves successful in the prediction of existence and stability of all the nonlinear modes considered. In certain regions of the parameter space, we find good mobility of localized excitations, an interesting feature which is not present in standard 2D-DNLS systems.

This article is organized as follows: In Section 2, the general model and the method employed to study it are introduced. Then, we present and discuss our results regarding the effect of dipolar interaction on the relation between Norm and eigen-energy (timeevolution factor), for different families of localized solutions. Also, the stability of these solutions is studied, leading to an observation of bi-unstable regions. The Norm thresholds properties are analyzed in detail. Section 3 presents the analysis of asymmetric stationary solutions which exist in the bi-unstable regions. We also study the dynamics of localized solutions. In Section 4, the system with attractive dipolar interactions is studied with the same formalism as in Sec. 2. Section 5, we discuss some approximations, which support analytical expressions around critical values of the interaction parameter. Finally, Section 6 concludes the paper

\section{Two-dimensional lattice with long-range nonlinear interactions}

The dynamics of the wave function amplitude $u_{i, j}$ at site $i, j$ of an isotropic square lattice is governed by a generalized DNLS equation

$$
\begin{aligned}
-i \dot{u}_{i, j}= & \kappa\left(u_{i+1, j}+u_{i-1, j}+u_{i, j+1}+u_{i, j-1}\right)+q\left|u_{i, j}\right|^{2} u_{i, j} \\
& +g\left(\left|u_{i+1, j}\right|^{2}+\left|u_{i-1, j}\right|^{2}+\left|u_{i, j+1}\right|^{2}+\left|u_{i, j-1}\right|^{2}\right) u_{i, j} .
\end{aligned}
$$

Here, $\kappa$ is the linear coupling coefficient, induced by the hopping of atoms between neighboring lattice sites. The strength of the onsite nonlinearity (proportional to the atomic scattering length) is quantified by parameter $q$. The nonlinear coupling $g$ describes the long-range dipolar interaction between atoms on adjacent sites.

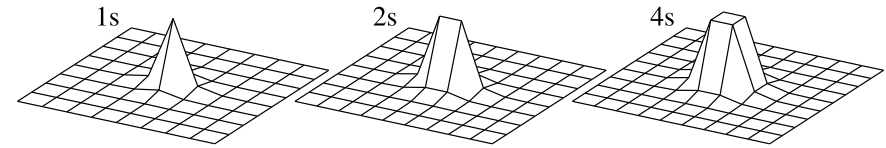

Fig. 1. Fundamental modes centered at different positions

These coefficients can be obtained from a continuum approach, as a direct extension of the 1D case [30].

Norm and stability of stationary solutions. Due to the presence of nonlinearity, the existence of localized solutions of model (1) is expected [19]. In this work, we will consider three types of fundamental localized modes: the on-site (one-peak, 1s) solution, centered at one site of the lattice; the inter-two-sites (two-peaks, $2 \mathrm{~s}$ ) solution, centered in between two sites having the same amplitude; and the inter-four-sites (four-peaks, 4s) solution, centered in between four sites having the same amplitude. Fig. 1 shows typical profiles for $1 \mathrm{~s}, 2 \mathrm{~s}$ and $4 \mathrm{~s}$ nonlinear modes.

We look for stationary solutions of the form $u_{i, j}(t)=u_{i, j} e^{i \lambda t}$ with $u_{i, j} \in \mathbb{R}$, where $\lambda$ represents the solution's energy. Substitution into eq. (1) leads to the following $n \times n$ algebraic system:

$$
\begin{aligned}
\lambda u_{i, j}= & \kappa\left(u_{i+1, j}+u_{i-1, j}+u_{i, j+1}+u_{i, j-1}\right)+q u_{i, j}^{3} \\
& +g\left(u_{i+1, j}^{2}+u_{i-1, j}^{2}+u_{i, j+1}^{2}+u_{i, j-1}^{2}\right) u_{i, j} .
\end{aligned}
$$

Linear coupling interactions define the single-particle energy spectrum for this 2D square lattice. By setting $q=g=0$, and solving model (2) using a plane wave ansatz, we obtain the following energy band: $\lambda=2 \kappa\left(\cos k_{x}+\cos k_{y}\right) . k_{x}$ and $k_{y}$ represents the horizontal and vertical quasimomentum. Therefore, the linear energies are bounded in the region $\lambda \in[-4 \kappa, 4 \kappa]$, that forms the linear spectrum of this square lattice. For a finite system of $n \times n$ sites, bounds reduce to $\pm 4 \kappa \cos (\pi /[n+1])$, which tends to $\pm 4 \kappa$ for $n \rightarrow \infty$.

In the following, without loss of generality, we set $\kappa=1$ and $q=1$ [this could be achieved as well by normalizing all parameters in model (1)]. System (2) is solved numerically by means of a standard Newton-Raphson method. We start at the regime of very high energy $\lambda$, where the number of particles (Norm) of the solution, defined as

$N=\sum_{i j}\left|u_{i j}\right|^{2}$

is expected to be large too (anti-continuum limit [6]). In this limit, coupling between sites can be neglected, and thus any profile, constructed from multiple single-site solutions, becomes a stationary solution of eq. (1). Decreasing $\lambda$ (and $N$ ) allows us to construct a whole family of fundamental localized solutions for given values of $q$ and $g$. We study different solution families by plotting $N$ vs $\lambda$ curves, as shown in Fig. 2(a), for the case $g=0$ and $g=0.3$ (considering an array of $15 \times 15$ lattice sites).

Apart from the atom number $N$, another conserved quantity of our system is the Hamiltonian

$$
\begin{aligned}
H & =-\sum_{i j}\left[\left(u_{i+1, j}+u_{i, j+1}\right) u_{i j}^{*}\right. \\
& \left.+\frac{g}{2}\left(\left|u_{i+1, j}\right|^{2}+\left|u_{i, j+1}\right|^{2}\right)\left|u_{i j}\right|^{2}+\text { c.c. }\right]-\frac{1}{2} \sum_{i j}\left|u_{i j}\right|^{4},
\end{aligned}
$$

where the first two terms represent the linear and nonlinear interactions for nearest-neighbors only, in a 2D square lattice. The Hamiltonian helps us to study the dynamical properties of the system when trying to move localized solutions across the lattice. A minimum (maximum) of $H$ is an indication of stable (unstable) solutions, which is an important consideration in order to effectively overcome the induced nonlinear dynamical barriers.
67 68 69 70 71 72 73 74 75 76 77 78 79 80 81 82 83 84 85 86 87 88 89 90 91 92 93 94 95 96 97 98 99 

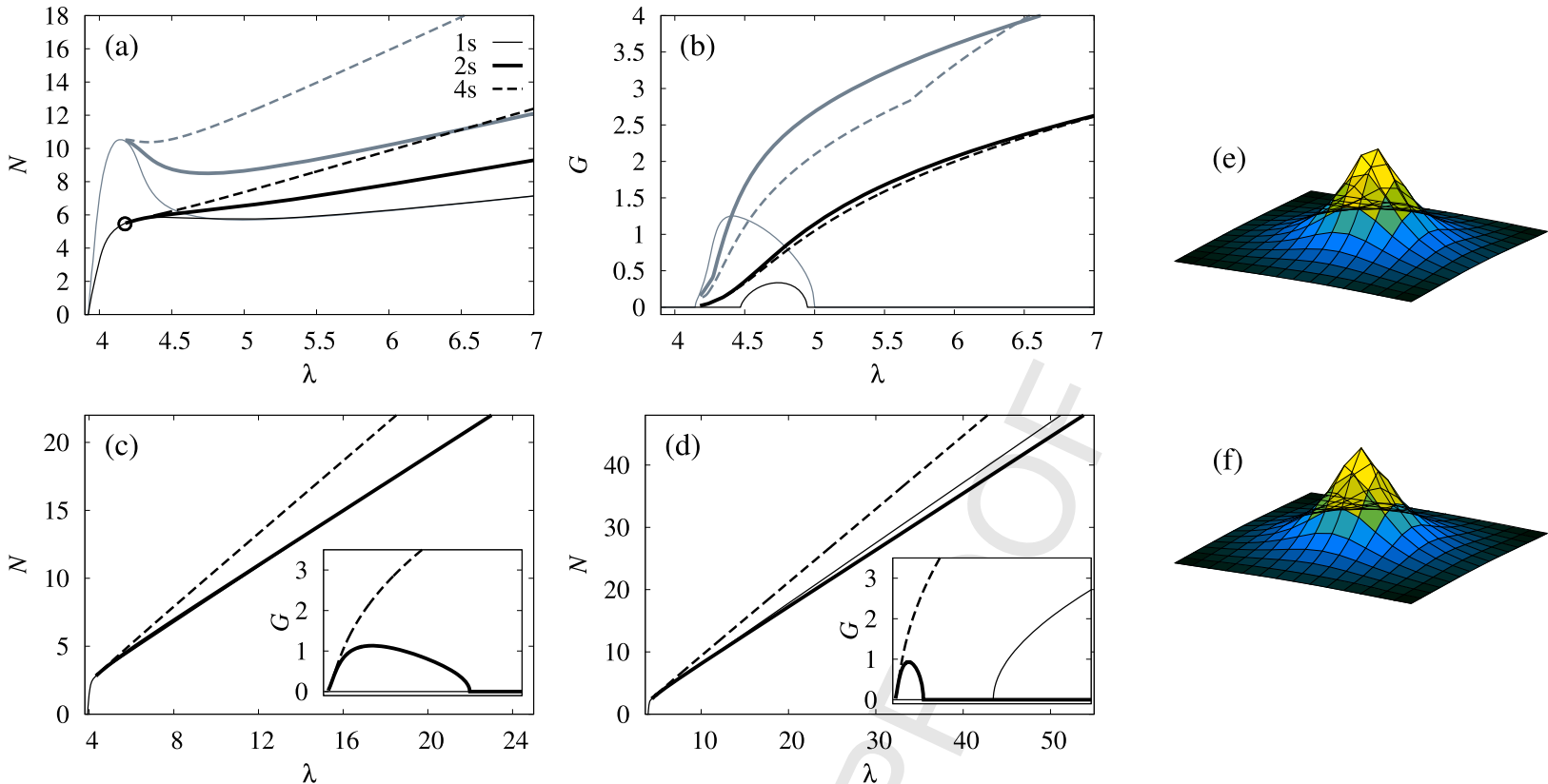

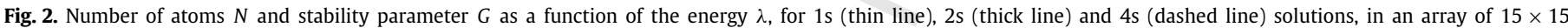

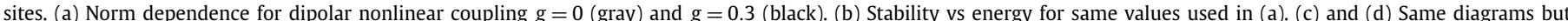

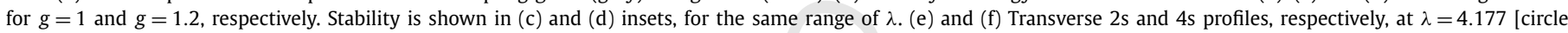
in (a)], near the edge of the linear band for $g=0.3$.

Once a stationary solution $\left\{u_{i j}\right\}$ is found, for a given value of $\lambda$, we compute its linear stability by implementing a standard method as presented, e.g., in Ref. [31]. Instability of a solution can be quantified by the gain $G$, which corresponds to the maximum complex eigenvalue. Only for $G=0$ solutions are stable. The dependence of $G$ on $\lambda$ for the cases $g=0$ and $g=0.3$ is shown in Fig. 2(b). Plots of Norm and Gain vs $\lambda$ obtained for two further values of the nonlinear interaction $g$ are shown in Figs. 2(c) and (d). Notice that, as we approach the solution's energy to the upper edge of the linear band (located at $4 \cos (\pi /[n+1])$ for an array of $n \times n$ sites), solutions get broader and start to become undistinguishable. We observe a fusion into a single curve for all solutions [see profiles in Fig. 2(e, f)]. This is because all these fundamental solutions bifurcate from the same in-phase linear fundamental mode at the upper edge of the linear spectrum.

Our results highlight another feature of the system near the linear band, namely, a change in the curvature of the $N$ vs $\lambda$ diagrams. It is widely known that in absence of dipolar interactions ( $g=0$, DNLS case) there exists an energy threshold [19], upon which localized solutions exist for infinite systems. This is normally associated with a local minimum for the Norm vs energy curve [27] (which also defines a change in the stability of the 1 s solution). Solutions from the localized branch occupy only few lattice sites, whereas the solutions bifurcating from the linear band edge are broad. Therefore, the connection between these two different nonlinear branches generates a minimum in the $N$ vs $\lambda$ curve. As $g$ increases we observe that this threshold tends to disappear in finite systems. Fig. 2, shows how this minimum still exists for a nonlinear coupling of $g=0.3$ but has already disappeared for $g=1$. Similar to the case of a 1D lattice [30], the increment of $g$ implies a smaller slope in the bifurcating branch. Therefore, for finite lattices, above some critical $g$-value, the connection between the low-Norm bifurcation regime and the largeNorm localized branch is now found to meet smoothly, without any intermediate minimum. Dipolar interactions broaden the nonlinear modes considerably due to the long-range nonlinear coupling, which helps to smoothen this transition.
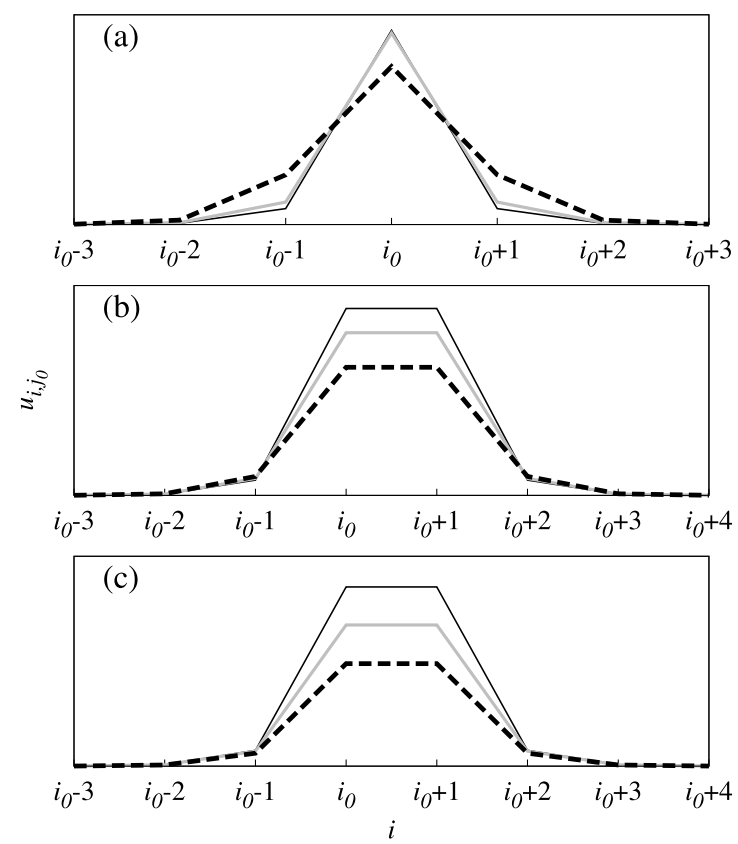

Fig. 3. Amplitudes at the center row of the (a) $1 \mathrm{~s}$, (b) $2 \mathrm{~s}$ and (c) $4 \mathrm{~s}$ solutions with energy $\lambda=12.7$. Thin black, thick gray and thick dashed lines correspond to dipolar nonlinear coupling $g=0, g=0.3$, and $g=1$, respectively. The change in the shape of the solutions for increasing $g$ is related to the variation of the curves in Fig. 2.

For larger values of Norm and energy (i.e., far from the linear band), the relation between these two quantities becomes approximately linear. We observe a decrease in the slope of the $N$ vs $\lambda$ curve for both the $2 \mathrm{~s}$ and $4 \mathrm{~s}$ solutions when $g$ grows. In fact, when $g=q=1$ [Fig. 2(c)], the $1 \mathrm{~s}$ and $2 \mathrm{~s}$ modes have the same slope and overlap for large $N$. For $g>q$, the asymptotic 2s curve is always below the 1s curve [Fig. 2(d)]. The behavior of the slope of the $N$ vs $\lambda$ curves is reflected in the profiles of the respective solutions. In Fig. 3 we show cuts for the three modes, corresponding 

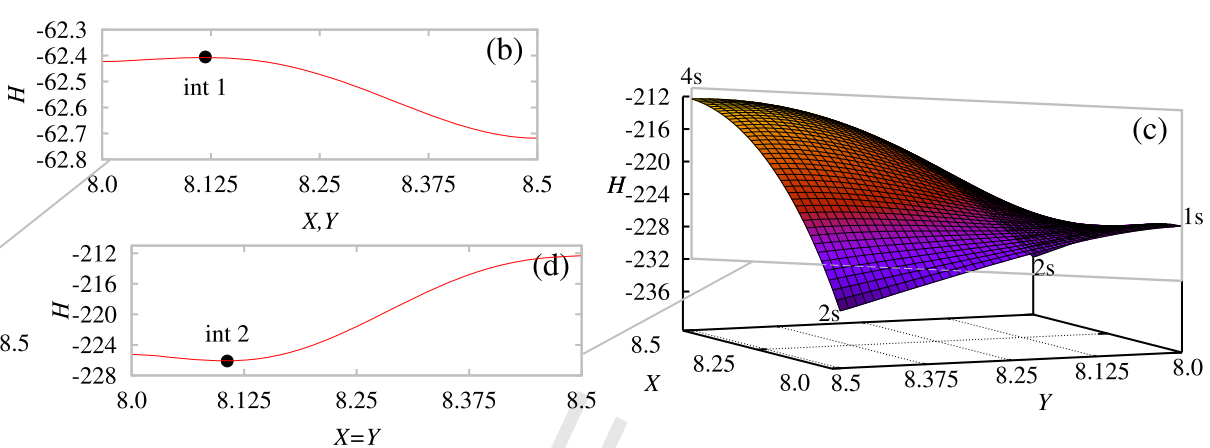

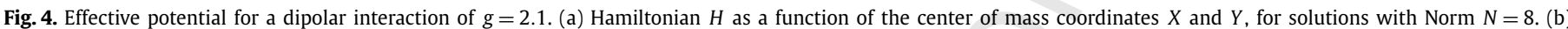

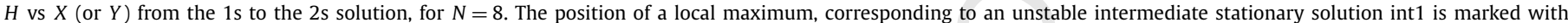

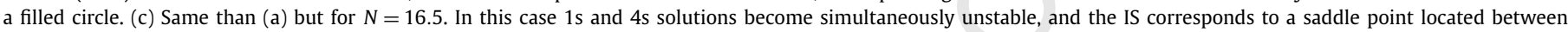
them, as shown in (d).
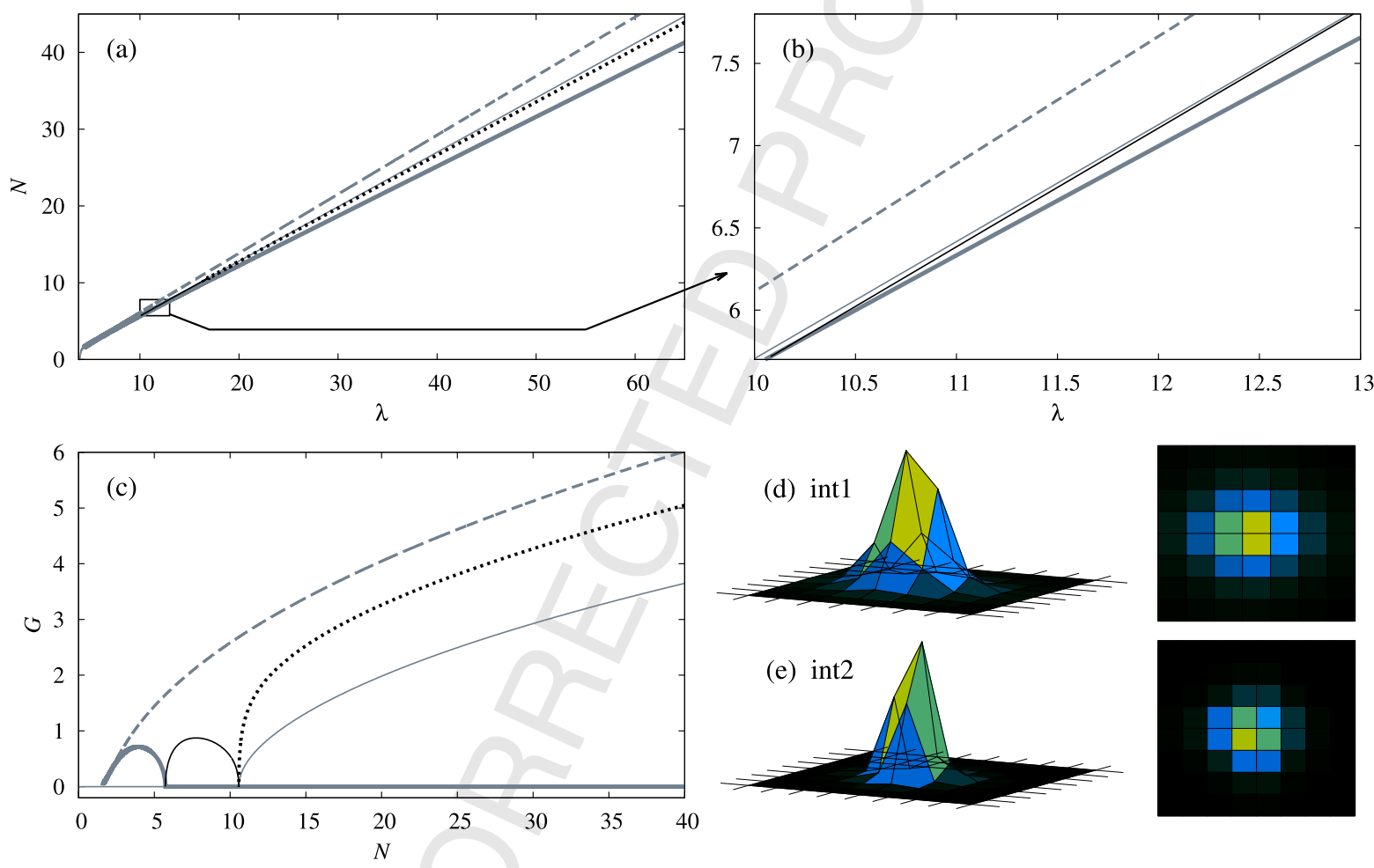

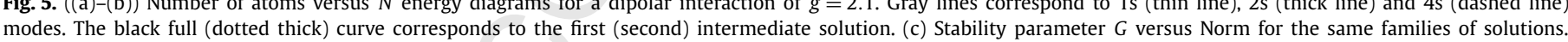

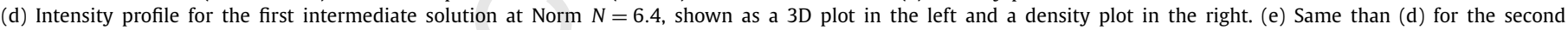
intermediate solution at Norm $N=33.9$.

to the 8-th row, for a fixed energy value and the same values of $g$ studied in Fig. 2. Notice how the 1s solution reduces its central peak and widens considerably, thus keeping a similar value of the Norm at the same $\lambda$. Instead, the $2 \mathrm{~s}$ and $4 \mathrm{~s}$ modes just reduce their overall amplitude, which is consistent with the decrease in the slope of their respective $N$ vs $\lambda$ curves. This transition is accompanied by a change on the stability of the 2 s solution, which becomes stable for larger values of the Norm, as shown in the insets of Figs. 2(c) and (d). When $g$ is above $q$, the 1 s solution is also stable in a range of parameters before becoming unstable [see inset of Fig. 2(d)]. Thus, a bi-stable region arises, which may imply the existence of intermediate solutions $[27,28,30]$.

\section{Intermediate solutions}

As a complement to the simultaneous stability of the $1 \mathrm{~s}$ and $2 \mathrm{~s}$ modes over a range of energy, we expect that there exists an un- stable intermediate solution (IS) centered between the $1 \mathrm{~s}$ and $2 \mathrm{~s}$ stable solutions. In order to find it, we implement the constraint method [32,33], which allows us to construct an effective energy potential, i.e., the Hamiltonian energy as a function of the center of mass $(X, Y) \equiv \sum_{i, j}(i, j)\left|u_{i, j}\right|^{2} / N$, for a fixed Norm $N$ (see Refs. $[28,34,30])$. Calculation is performed over a square area, at the central region of a $15 \times 15$ array. In this context, the coordinates $(8,8)$ correspond to $1 \mathrm{~s},(8.5,8)$ and $(8,8.5)$ to horizontal and vertical $2 \mathrm{~s}$, respectively, and $(8.5,8.5)$ to 4 s solutions. In the bi-stable region, the unstable intermediate solution (denoted as int1) maximizes the effective potential, while the $1 \mathrm{~s}$ and $2 \mathrm{~s}$ solutions are local minima of this energy landscape. This is depicted in Fig. 4(a) and (b), where we show the effective potential for solutions with Norm $N=8$, and a dipolar interaction of $g=2.1$. As can be seen, there is a local maximum between the $1 \mathrm{~s}$ and $2 \mathrm{~s}$ solutions (filled circle). Once an IS has been found, we can use it as a seed to construct the respective Norm vs energy curve, and to compute its stability. This 
way, we obtain the curves shown in Fig. 5, where we have included the respective results for the $1 \mathrm{~s}, 2 \mathrm{~s}$ and $4 \mathrm{~s}$ modes for the sake of comparison. The maximum eigenvalue $G$ of the stability matrix is now plotted as a function of the Norm $N$, in order to make clearer its effect on the dynamics of localized solutions (studied below), which involves stationary solutions at a fixed Norm [29]. From the point where the $1 \mathrm{~s}$ solution becomes unstable $(N \approx 11)$, a second IS is found (dotted line in Fig. 5). This mode is centered between the $1 \mathrm{~s}$ and $4 \mathrm{~s}$ solutions, so it corresponds to a saddle point in the effective potential [see Fig. 4(c) and (d) for $N=16.5$ ]. However, since no further stability exchanges occur for larger $N$, this intermediate mode does not join the $1 \mathrm{~s}$ and $4 \mathrm{~s} N-\lambda$ curves, in contrast to what occurs at the bi-stable range.

Dynamics of localized excitations. To force an arbitrary localized stationary profile $\left\{u_{n, m}\right\}$ to move across the lattice, we may add a certain amount of kinetic energy (kick). Therefore, we multiply the amplitude $u_{n, m}$ by $\exp \left[i\left(k_{x} n+k_{y} m\right)\right]$ and use this as an initial condition to numerically integrate Eq. (1). Physically, the introduction of this phase gradient between sites implies to give a transversal momentum to a BEC wave-function or, equivalently, to accelerate the lattice. ${ }^{1}$

Fixing the dipolar interaction parameter at $g=2.1$, we notice that the $4 \mathrm{~s}$ solution is always unstable for any $N$, with a $G$-value always greater than those for the $1 \mathrm{~s}$ and $2 \mathrm{~s}$ solutions. The condition of instability may promote mobility of the $4 \mathrm{~s}$ solution, as the effective potential surfaces shown in Fig. 4 suggest: Unstable solutions are local maxima of the pseudo-potential; therefore, by adding a small kick the solution may start to move. When the perturbation is strong or the kick direction does not correspond to any fundamental solution in the potential landscape, the profile may decay into some other localized states and/or radiation modes. In contrast to the usual cubic DNLS case, the present model may allow the mobility of very localized solutions. We show two examples in Fig. 6, where the center of mass of a localized profile is plotted versus time. Indeed, profiles keep well localized in space while moving across the lattice, as can be seen in this figure. Notice that as the solution travels across the lattice, transitioning from one stationary mode to another, its center of mass accelerates or decelerates according to the position of the modes in the potential landscape and their respective stability.

Since the existence of intermediate solutions modifies the Peierls-Nabarro barrier (defined as a Hamiltonian difference between two fundamental stationary solutions [25]), there is also an effect on the transversal evolution of localized excitations. In our case, the existence of unstable intermediate solutions hinders the mobility. In contrast to saturable-DNLS lattices [28], we were not able to find mobility windows in the vicinity of the second (superior) bifurcation point. We observe that both, the int 1 and int2 modes are always very unstable, what is an indication that the effective barrier is too large and the mobility is forbidden. Thus, transport of localized excitations is found ${ }^{2}$ only for low Norm regions (below the range of existence of intermediate solutions). The dipolar nonlinear interaction allows for the broadening of modes, and it contributes to the exchange of their stability properties and to the corresponding mobility presented in Fig. 6.

\section{Attractive dipolar interaction}

Now we consider negative values of the dipolar coefficient $g$ in our model, corresponding to attractive dipolar interaction. Regarding the parameters treated before (Norm and stability Gain), we

\footnotetext{
1 Cfr. section IV of [17].

2 To identify possible mobility windows, we performed several dynamical simulations using a fine tuning of the quasimomentum and Norm $N$.
}
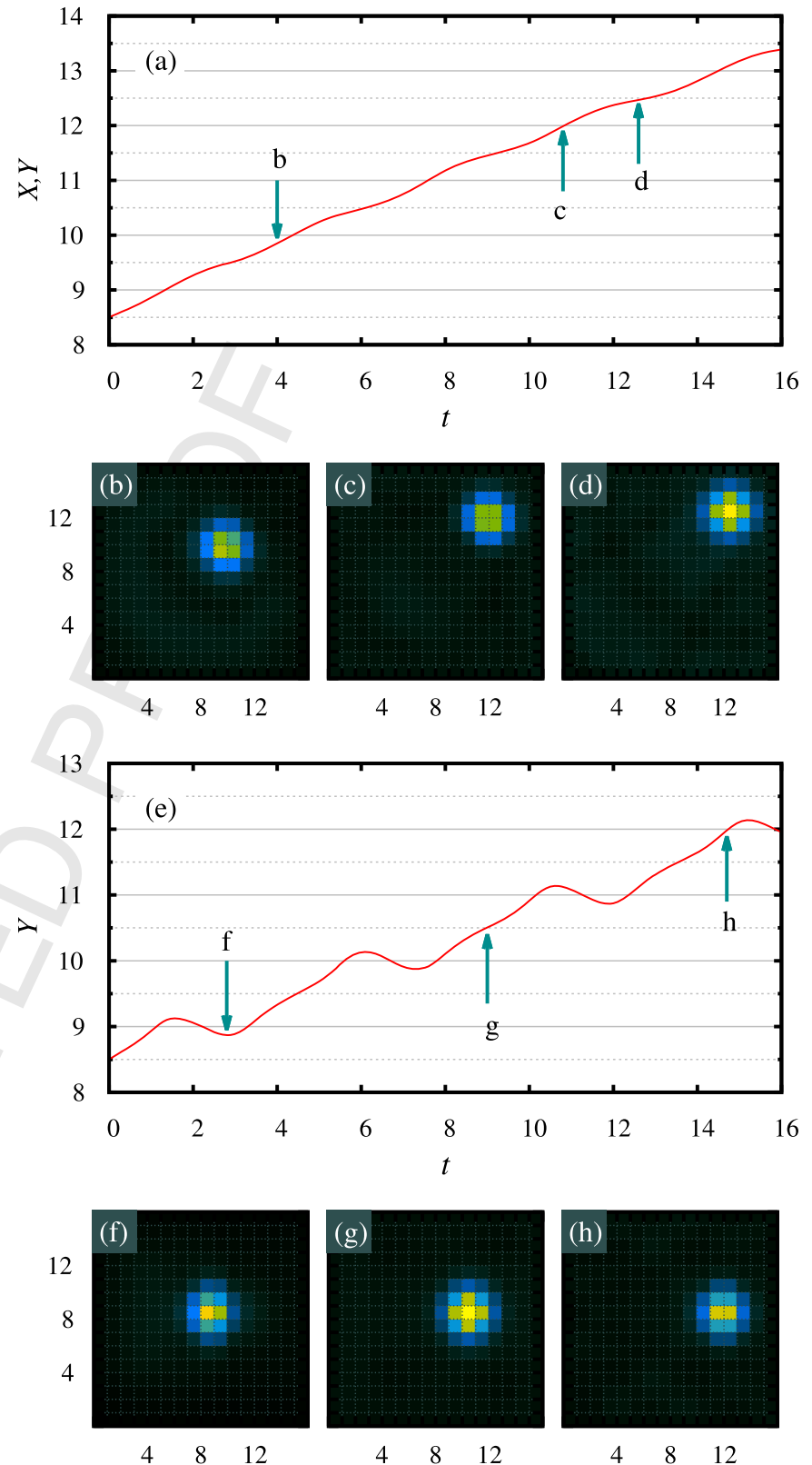

Fig. 6. Dynamics of localized solutions on a $15 \times 15$ array for $g=2.1$. (a) Evolution of the center of mass of the excitation when a kick $k_{x}=k_{y}=0.35$ is applied to a $4 \mathrm{~s}$ solution with Norm $N=4.8$. Plots (b)-(d) show the spatial profile at certain times indicated in (a). (e) Evolution of the $Y$ component of the center of mass when a kick $k_{y}=0.3$ is applied to a $4 \mathrm{~s}$ mode with Norm $N=5.8$. Plots (f)-(h) show the spatial profile at certain times indicated in (e). Notice how the speed of the center of mass changes as the profile transits between the stationary solutions, whose center of mass is indicated with horizontal lines in (a) and (e).

see that a null dipolar interaction $g=0$ does not correspond to a critical point, but rather the behavior described previously is initially continued for $g<0$. Thus, the asymptotic slope of the Norm vs energy curve for $2 \mathrm{~s}$ and $4 \mathrm{~s}$ solutions increases for lower $g$. The 1s family still exhibits a minimum Norm before joining the other solutions when decaying into the linear band, as shown in Fig. 7(a) for $g=-0.05$.

However, as we approach the value $g \rightarrow-0.25$, we observe that solutions near the linear band exhibit a special $N$ vs $\lambda$ dependence: the final decay of the Norm, before joining the linear band, is now very abrupt and occurs very close to the upper band limit, so all solutions tend to enter into the band at a finite Norm value, while becoming unstable [see Fig. 7(b)]. Indeed, once the

\section{0}

81

82 


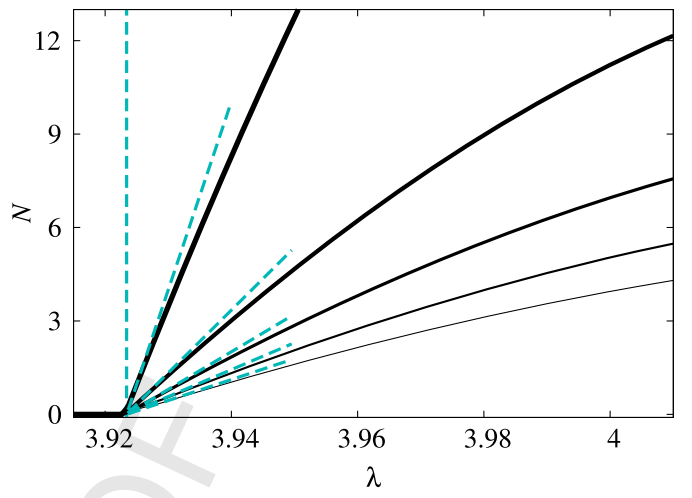

Fig. 8. $N$ vs $\lambda$ curve near the upper edge of the linear band. From thiner to thicker lines the value of $g$ is $0.2,0.1,0,-0.1$, and -0.2 . Dashed lines correspond to the fit obtained using Eq. (5). The vertical line corresponds to $g=-0.25$, depicting the divergence of the slope in Eq. (5); in this case the number of particles $N$ does not tend to zero when energy reaches the band edge.

$N \sim n^{2} A^{2}=\frac{n^{2}(\lambda-4 \kappa)}{(q+4 g)}$,

Fig. 7. (a)-(d) $N$ vs $\lambda$ curves for attractive dipolar interaction $g=-0.05,-0.2$ energy. In all the graphs, the vertical blue dotted line marks the upper edge of the linear band. (e)-(g) Intensity profiles of solutions at marked points. (For interpretation of the references to color in this figure legend, the reader is referred to the web version of this article.)

value $g=-0.25$ is reached [Fig. 7(c)], a discontinuity appears in the $N$ vs $\lambda$ curve of the $1 \mathrm{~s}$ solution, while the Norm of $2 \mathrm{~s}$ and $4 \mathrm{~s}$ fundamental modes does not go to zero anymore, and actually diverges at the edge of the linear band. For $g<-0.25$, all curves are continued into the linear spectrum of eigen-energies as families of solutions whose Norm increases rapidly while decreasing the solution energy [Fig. 7(d)]. From this value, all solutions get a Norm threshold, originated when a decaying curve (coming from inside the linear band) connects to an increasing curve (coming from the gap). In Ref. [30] a similar result was obtained for a 1D system, where the critical value of the normalized parameter of nonlinear interaction was -0.5 . In that work, the existence of nonlinear solutions (i.e. high Norm solutions) with energies within the linear band was related to the stability of nonlinear uniform plane waves below the critical value, i.e. the absence of modulational instability (MI) in the $g<g_{\text {crit }}$ regime. Since MI facilitates the appearance of nonlinear localized modes, it is reasonable to think that its absence hinders the appearance of localized solutions inside the linear band. Indeed, in our case, when $g<-0.25$, the families of localized nonlinear modes does not bifurcate from the linear band anymore: modes around the upper edge of the band do not exhibit an extended low Norm profile as in the $g>-0.25$ case [compare Figs. 7(e) and (f)], but rather resemble a superposition of a localized solution with an uniform extended background.

\section{Analytical approximations}

In order to characterize the asymptotic dependence of the Norm with respect to the energy, for the localized solutions, we use an approximation developed in Ref. [30]. For any value of the Norm $N$, the $1 \mathrm{~s}$ solution corresponds to a single peak excitation of amplitude $A$, centered at the site $\left(i_{0}, j_{0}\right)$, and surrounded by four smaller amplitudes at the neighboring sites, i.e.

$u_{i, j}=A\left[\delta_{i, i_{0}} \delta_{j, j_{0}}+\alpha\left(\delta_{i, i_{0} \pm 1} \delta_{j, j_{0}}+\delta_{i, i_{0}} \delta_{j, j_{0} \pm 1}\right)\right]$,

with $\alpha<1$. Substitution of this Ansatz on Eqs. (2) for the central site leads to $A^{2}=(\lambda-4 \alpha \kappa) /\left(q+4 g \alpha^{2}\right)$. As $N$ is reduced and $\lambda$ gets closer to the linear band, the solution widens, so we can take the limit $\alpha \rightarrow 1$, getting with $n^{2}$ the size of the array. From this expression, it is clear that the rate at which $N$ increases, for energies slightly above the linear band, grows and becomes indeterminate as $g$ tends to $-q / 4$, as it was shown from our numerical computations in the previous section. Fig. 8 depicts the bifurcation from the linear band for four different values of $g$ near $-q / 4$, showing good agreement between approximation (5) and numerical results. This estimation also gives us an insight for the phenomenology observed inside the linear band. For $g<-0.25 q$, the denominator in (5) gets negative. As Norm $N$ is defined positive, this implies that solutions close to the linear band $(\alpha \approx 1)$ possess a frequency which is smaller than $4 \kappa$, therefore existing inside the linear band region [see Fig. 7(d)].

On the other hand, far from the linear band, when $\lambda$ and $N$ increase the solution becomes more localized, so the approximation $\alpha \rightarrow 0$ can be used. Then,

$N_{1 \mathrm{~s}} \simeq A^{2} \simeq \frac{\lambda}{q}$

By repeating the same analysis for the 2 s solution, and considering the same large Norm limit, we obtain

$N_{2 s} \simeq 2 A^{2} \simeq \frac{2(\lambda-\kappa)}{q+g}$.

Both expressions are in agreement with the numerical calculations for large $N$ (and $\lambda$ ) for $g \leq q$. Eq. (7) sheds light on the decrease in the slope of the $N$ vs $\lambda$ curve for the 2 s solution. Also, it correctly predicts that for $g=q$, both $1 \mathrm{~s}$ and $2 \mathrm{~s}$ solutions will experience the same asymptotic dependence of $N$ on $\lambda$, as we showed previously in Fig. 2(c). For $g>q$ we observe that this dependence changes and the curve of the $2 \mathrm{~s}$ solutions possesses a smaller slope than the $1 \mathrm{~s}$ solution, effectively changing the phenomenology and stability properties. This clearly shows that a dipolar nonlinear coupling coefficient effectively changes the dynamics on 2D square lattices.

\section{Conclusions}

We have studied the effect of long-range dipolar interactions on the properties of a BEC loaded into a periodic optical lattice, in the context of discrete nonlinear systems. As the nonlinear and nonlocal dipolar interaction between sites is increased, a notable variation in the number of atoms vs energy relation is observed, which also manifests as a change in the stability properties of 
some fundamental solutions. Furthermore, we observe the disappearance of the Norm threshold of localized modes in finite lattices, when the nonlinear interaction crosses a critical value. The existence of bi-unstable regions, i.e. ranges of energies where two different localized solutions are simultaneously unstable, allows for the existence of intermediate solutions. Also, we observe that the long-range dipolar interaction (quantified by the parameter $g$ ) allows mobility of well localized excitations with low Norm, which is not possible in standard two-dimensional DNLS systems. For $g<-q / 4$ (being $q$ the on-site nonlinear interaction of the DNLS model), we found that solutions always have a Norm threshold when approaching the linear band and, then, a fast linear increment of the Norm for frequencies below the upper band edge. As a consequence, localized mode profiles acquire an important background that increases homogeneously in amplitude as their Norm increases.

\section{Acknowledgements}

The authors wish to thank Programa ICM grant RC130001, the Spanish government project FIS 2011-25167, FPDI-2013-18422 as well as the Aragón project (Grupo FENOL), and FONDECYT Grants No. 1151444 and No. 1140635.

\section{References}

[1] A. Griesmaier, J. Werner, S. Hensler, J. Stuhler, T. Pfau, Bose-Einstein condensation of chromium, Phys. Rev. Lett. 94 (2005) 160401.

[2] M. Lu, S.H. Youn, B.L. Lev, Trapping ultracold dysprosium: a highly magnetic gas for dipolar physics, Phys. Rev. Lett. 104 (2010) 063001.

[3] M. Lu, N.Q. Burdick, S.H. Youn, B.L. Lev, Strongly dipolar Bose-Einstein condensate of dysprosium, Phys. Rev. Lett. 107 (2011) 190401.

[4] P. Pedri, L. Santos, Two-dimensional bright solitons in dipolar Bose-Einstein condensates, Phys. Rev. Lett. 95 (2005) 200404.

[5] I. Tikhonenkov, B. Malomed, A. Vardi, Anisotropic solitons in dipolar BoseEinstein condensates, Phys. Rev. Lett. 100 (2008) 090406.

[6] R. MacKay, S. Aubry, Proof of existence of breathers for time-reversible or Hamiltonian networks of weakly coupled oscillators, Nonlinearity 7 (1994) 1623.

[7] G.-P. Zheng, Solitons of dipolar spinor condensates in an optical lattice, J. Phys. B 40 (2007) 4493.

[8] G. Gligoríc, A. Maluckov, L. Hadzievski, B.A. Malomed, Bright solitons in the one-dimensional discrete Gross-Pitaevskii equation with dipole-dipole interactions, Phys. Rev. A 78 (2008) 063615.

[9] G. Gligoríc, A. Maluckov, L. Hadzievski, B.A. Malomed, Soliton stability and collapse in the discrete nonpolynomial Schrödinger equation with dipole-dipole interactions, Phys. Rev. A 79 (2009) 053609.

[10] G. Gligoríc, A. Maluckov, L. Hadzievski, B.A. Malomed, Collapse instability of solitons in the nonpolynomial Schrödinger equation with dipole-dipole interactions, J. Phys. B 42 (2009) 145302.

[11] J. Cuevas, B.A. Malomed, P.G. Kevrekidis, D.J. Frantzeskakis, Solitons in quasione-dimensional Bose-Einstein condensates with competing dipolar and local interactions, Phys. Rev. A 79 (2009) 053608.
[12] B.B. Baizakov, F.K. Abdullaev, B.A. Malomed, M. Salerno, Solitons in the TonksGirardeau gas with dipolar interactions, J. Phys. B 42 (2009) 175302.

[13] L.E. Young, P. Muruganandam, S.K. Adhikari, Dynamics of quasi-onedimensional bright and vortex solitons of a dipolar Bose-Einstein condensate with repulsive atomic interaction, J. Phys. B 44 (2011) 101001.

[14] G. Gligoríc, A. Maluckov, M. Stepíc, L. Hadzievski, B.A. Malomed, Twodimensional discrete solitons in dipolar Bose-Einstein condensates, Phys. Rev. A 81 (2010) 013633.

[15] Z.-K. Lu, Y. Li, D.S. Petrov, G.V. Shlyapnkikov, Stable dilute supersolid of twodimensional dipolar bosons, Phys. Rev. Lett. 115 (2015) 075303.

[16] P. Köberle, D. Zajec, G. Wunner, B.A. Malomed, Creating two-dimensional bright solitons in dipolar Bose-Einstein condensates, Phys. Rev. A 85 (2012) 023630.

[17] O. Morsh, M. Oberthaler, Dynamics of Bose-Einstein condensates in optical lattices, Rev. Mod. Phys. 78 (2006) 179.

[18] Y.V. Kartashov, B.A. Malomed, L. Torner, Solitons in nonlinear lattices, Rev. Mod. Phys. 83 (2011) 247.

[19] S. Flach, K. Kladko, R.S. MacKay, Energy thresholds for discrete breathers in one-, two-, and three-dimensional lattices, Phys. Rev. Lett. 78 (1997) 1207.

[20] G. Kalosakas, K.O. Rasmussen, A.R. Bishop, Delocalizing transition of BoseEinstein condensates in optical lattices, Phys. Rev. Lett. 89 (2002) 030402.

[21] P.L. Christiansen, Y.B. Gaididei, V.K. Mezentsev, S.L. Musher, K.O. Rasmussen, J.J. Rasmussen, I.V. Ryzhenkova, S.K. Turitsyn, Discrete localized states and localization dynamics in discrete nonlinear Schrödinger equations, Phys. Scr. T 67 (1996) 160.

[22] P.L. Christiansen, Y.B. Gaididei, M. Johansson, K.O. Rasmussen, Solitary excitations in discrete two-dimensional nonlinear Schrödinger models with dispersive dipole-dipole interactions, Phys. Rev. B 57 (1998) 11303.

[23] P.L. Christiansen, Y.B. Gaididei, K.O. Rasmussen, V.K. Mezentsev, J.J. Rasmussen, Dynamics in discrete two-dimensional nonlinear Schrödinger equations in the presence of point defects, Phys. Rev. B 54 (1996) 900.

[24] E. Arévalo, Soliton theory of two-dimensional lattices: the discrete nonlinear Schrödinger equation, Phys. Rev. Lett. 102 (2009) 224102.

[25] Y.S. Kivshar, D.K. Campbell, Peierls-Nabarro potential barrier for highly localized nonlinear modes, Phys. Rev. E 48 (1993) 3077.

[26] C. Mejía-Cortés, R.A. Vicencio, B.A. Malomed, Mobility of solitons in onedimensional lattices with the cubic-quintic nonlinearity, Phys. Rev. E 88 (2013) 052901.

[27] R.A. Vicencio, M. Johansson, Discrete soliton mobility in two-dimensional waveguide arrays with saturable nonlinearity, Phys. Rev. E 73 (2006) 046602.

[28] U. Naether, R.A. Vicencio, M. Johansson, Peierls-Nabarro energy surfaces and directional mobility of discrete solitons in two-dimensional saturable nonlinear Schrödinger lattices, Phys. Rev. E 83 (2011) 036601.

[29] M. Johansson, P. Jason, Breather mobility and the Peierls-Nabarro potential: brief review and recent progress, Springer Series in Materials Science 221 (2015) 147

[30] S. Rojas-Rojas, R.A. Vicencio, M.I. Molina, F.K. Abdullaev, Nonlinear localized modes in dipolar Bose-Einstein condensates in optical lattices, Phys. Rev. A 84 (2011) 033621.

[31] A. Khare, K.O. Rasmussen, M.R. Samuelsen, A. Saxena, Exact solutions of the saturable discrete nonlinear Schrödinger equation, J. Phys. A 38 (2005) 807.

[32] M.I. Molina, R.A. Vicencio, Y.S. Kivshar, Discrete solitons and nonlinear surface modes in semi-infinite waveguide arrays, Opt. Lett. 31 (2006) 1693.

[33] C.R. Rosberg, D.N. Neshev, W. Krolikowski, A. Mitchell, R.A. Vicencio, M.I. Molina, Y.S. Kivshar, Observation of surface gap solitons in semi-infinite waveguide arrays, Phys. Rev. Lett. 97 (2006) 083901.

[34] U. Naether, R.A. Vicencio, M. Stepić, Mobility of high-power solitons in saturable nonlinear photonic lattices, Opt. Lett. 36 (2011) 1467. 